OPEN ACCESS

Edited by:

Andrew T. Olagunju,

McMaster University, Canada

Reviewed by:

Erich Flammer,

ZfP Südwürttemberg, Germany

Adegboyega Ogunwale, Neuropsychiatric Hospital, Aro,

Abeokuta, Nigeria

*Correspondence:

Marie-Josée Fleury

flemar@douglas.mcgill.ca

Specialty section:

This article was submitted to

Public Mental Health,

a section of the journal

Frontiers in Psychiatry

Received: 01 July 2021 Accepted: 01 November 2021 Published: 22 November 2021

Citation:

Gentil L, Grenier G, Meng X and

Fleury M-J (2021) Impact of Co-occurring Mental Disorders and

Chronic Physical Illnesses on Frequency of Emergency Department Use and Hospitalization for Mental Health Reasons.

Front. Psychiatry 12:735005 doi: 10.3389/fpsyt.2021.735005

\section{Impact of Co-occurring Mental Disorders and Chronic Physical Illnesses on Frequency of Emergency Department Use and Hospitalization for Mental Health Reasons}

\author{
Lia Gentil ${ }^{1,2}$, Guy Grenier ${ }^{1}$, Xiangfei Meng ${ }^{1,2}$ and Marie-Josée Fleury ${ }^{1,2 *}$ \\ ${ }^{1}$ Douglas Mental Health University Institute Research Centre, Montréal, QC, Canada, ${ }^{2}$ Department of Psychiatry, McGill \\ University, Montréal, QC, Canada
}

Background: Patients with mental disorders (MD) are at high risk for a wide range of chronic physical illnesses (CPI), often resulting in greater use of acute care services. This study estimated risk of emergency department (ED) use and hospitalization for mental health $(\mathrm{MH})$ reasons among 678 patients with MD and CPI compared to 1,999 patients with MD only.

Methods: Patients visiting one of six Quebec (Canada) ED for MH reasons and at onset of a MD in 2014-15 (index year) were included. Negative binomial models comparing the two groups estimated risk of ED use and hospitalization at 12-month follow-up to index ED visit, controlling for clinical, sociodemographic, and service use variables.

Results: Patients with MD, more severe overall clinical conditions and those who received more intensive specialized $\mathrm{MH}$ care had higher risks of frequent $\mathrm{ED}$ use and hospitalization. Continuity of medical care protected against both ED use and hospitalization, while general practitioner (GP) consultations protected against hospitalization only. Patients aged $65+$ had lower risk of ED use, whereas risk of hospitalization was higher for the 45-64- vs. 12-24-year age groups, and for men vs. women.

Conclusion: Strategies including assertive community treatment, intensive case management, integrated co-occurring treatment, home treatment, and shared care may improve adequacy of care for patients with MD-CPI, as well as those with MD only whose clinical profiles were severe. Prevention and outreach strategies may also be promoted, especially among men and older age groups.

Keywords: co-occurring mental disorders, chronic physical illnesses, emergency department (ED) use, hospitalization, clinical variables, sociodemographic variables, service use variables 


\section{INTRODUCTION}

Emergency department (ED) and inpatient hospital services are among the costliest forms of healthcare $(1,2)$, often serving as a barometer for the quality of healthcare systems (3-5). Frequent ED use or hospitalization may reflect poor access, continuity, or inappropriate outpatient care $(3,6)$. Patients with mental disorders (MD) including substance-related disorders (SRD) and with chronic physical illnesses (CPI) are high ED users, which contributes substantially to ED overcrowding (7-9). They are also hospitalized and readmitted more often than patients without MD or CPI (10-13). Co-occurring CPI frequently occurs among patients with MD (14-18). Mental disorders-chronic physical illnesses are associated with patient disability (19), social dysfunction (20), treatment complications (21), poor quality of life (19), and higher risk of mortality (22). Patients with MDCPI also face challenges in seeking care $(6,23)$, as healthcare systems tend to favor treatment of individual diseases rather than multimorbidity, whereas treating $\mathrm{MD}$ and CPI in isolation is generally ineffective $(24,25)$. As well, CPI in patients with MD are often under-diagnosed or undertreated $(18,23)$, particularly among patients with serious MD-CPI making limited use of primary care $(23,26,27)$. Other care-related challenges involve socio-economic barriers (28) and social stigma (29). General practitioners (GP) are usually viewed as inadequately equipped to treat more serious or complex $\operatorname{MD}(28,30)$.

The overall, combined effect of acute care service use for mental health $(\mathrm{MH})$ reasons among patients with $\mathrm{MD}$ and $\mathrm{CPI}$, compared with MD only, has rarely been assessed. Studies have more often investigated ED use and hospitalization for medical reasons. Of the few studies that compared MD-CPI with MD or CPI only, most found that patients with MD-CPI were more likely than those with CPI or MD only to use ED frequently (3133), to require hospitalization (34) and to face longer duration of hospital stay (32). Moreover, numerous studies have compared ED use and hospitalization for $\mathrm{MH}$ reasons, but only in terms of specific CPI and $\operatorname{MD}(31,33,35)$, e.g., diabetes and schizophrenia or serious $\operatorname{MD}(31,33,35)$, cancer and common MD (32), epilepsy and MD (36), migraine and MD (34), and CPI and MD among self-harming adolescents (25).

Most studies comparing patients with MD-CPI to those with MD or CPI only have controlled for few clinical and sociodemographic variables $(18,25,33-37)$. Results showed that the risk of frequent ED use and hospitalization increased with severity of CPI in patients with serious $\operatorname{MD}(24,33)$ or depressive disorders (18). Being a woman, younger and living in poorer neighborhoods reportedly increased ED use among patients with co-occurring schizophrenia and diabetes (31). Overall, this literature tended to omit assessments of service use variables,

Abbreviations: AIC, Akaike's Information Criterion; BIC, Bayesian Information Criterion; BDCU, emergency department database (Banque de Données Commune des Urgences); ED, emergency departments; GP, general practitioners; ICC, Intraclass Correlation Coefficient; ICD, International Classification of Diseases; MED-ECHO, hospitalization database (Maintenance et Exploitation des Données pour l'Étude de la Clientèle Hospitalière); MD, mental disorders; SRD, substancerelated disorders; RAMQ, Quebec health insurance regime (Régie de l'Assurance Maladie du Québec). although some studies did find that patients with MD-CPI and more frequent ED users were also more likely to use outpatient services, take medications $(34,38)$ and to be covered by health insurance (36).

Better knowledge of how MD-CPI, vs. MD only, impact $\mathrm{ED}$ use and hospitalization for $\mathrm{MH}$ reasons, controlling for service use, may contribute to improvement in health service management (39). Adequate intensity, continuity, and diversity of care for patients with CPI or MD and access to a family doctor or psychiatrist, particularly among patients with MD-CPI, could lower rates of ED use or preventable hospitalization. This study aimed to compare frequencies of ED use and hospitalization for $\mathrm{MH}$ reasons among patients with MD only compared to patients with MD-CPI, controlling for clinical, sociodemographic, and service use variables. We hypothesized that patients with MDCPI would make more frequent use of ED and experience more frequent hospitalization for $\mathrm{MH}$ reasons, and that both (1) severity of MD-CPI and (2) less intensity, diversity, or continuity of service use would increase frequency of ED use and hospitalization.

\section{METHODS}

\section{Study Context}

This study was conducted in the province of Quebec, which accounts for $22 \%$ of the Canadian population. Responsibility for Quebec health and social services is integrated within a single ministry. $\mathrm{MH}$ is one of nine service programs under the Quebec Ministère de la Santé et des Services sociaux (Ministry of Health and Social Services) (40). A major health reform in 2015 led to the creation of 22 integrated health and social services centers resulting from the merger of nearly all health and social institutions, like hospitals, community healthcare centers, and nursing homes, within each of the networks. Specialized MH services in the integrated networks are offered in psychiatric departments of general hospitals or in psychiatric hospitals. Public primary care $\mathrm{MH}$ services are offered by GP working in private medical clinics, in most cases family medicine groups. Over 60\% of Quebec GP work in these group practices, benefitting from added psychosocial clinicians like nurses and social workers and enhanced secretarial support. Family medicine groups insure patient registration, better access to care, and care continuity through expanded days and hours of medical coverage, including walk-in clinics (41). Primary $\mathrm{MH}$ care is also provided in community healthcare centers offering mainly psychosocial interventions, although some GP also work there on a salaried basis, whereas most physicians are remunerated on a fee-for-service basis. Community $\mathrm{MH}$ organizations (e.g., crisis centers, self-help groups) and psychologists working in private practice complete the Quebec MH system.

\section{Study Population and Design}

This 4-year cohort study (2012-13 to 2015-16) investigated 12,000 patients identified through provincial medical administrative databases who visited at least one of six Quebec ED in 2014-15 (index year) for MH reasons, including SRD. Only patients at onset of a MD at index ED visit, i.e., incident 
cases not diagnosed with MD in the previous 2 years, were included in the study $(n=2,819)$. Of these patients, 142 who were diagnosed with incident CPI after the date of their index ED visit in 2014-2015 (90 participants) were excluded from the study, as were those hospitalized for more than 90 days following the index ED visit (42), because outpatient care for these patients over the 12-month follow-up period would not be adequately assessed. The final sample thus consisted of 2,677 patients, $12+$ years old and eligible for health insurance under the Quebec health care regime [Régie de l'Assurance Maladie du Québec (RAMQ)]. Patients were further divided into two groups: those with MD-CPI $(n=678: 25 \%)$ or MD only ( $n$ $=1,999: 75 \%)$. Chronic physical illnesses were identified for a 2 -year period preceding index ED visits in 2014-15, at which time incident cases were diagnosed (43). Six ED located in major Quebec cities within University or peripheral health regions were selected for the study. The Quebec Access to Information Commission authorized the study and the ethics committee of a MH University institute approved the study protocol.

\section{Data Sources}

Data for the study were obtained from the RAMQ database, which contains Quebec medical administrative data including billing files for medical services provided by physicians on a fee-for-service basis, patient diagnoses, and patient sociodemographic information including material and social deprivation indices. Only $6 \%$ of billing occurred outside the public system in 2016-17 (RAMQ, 2020). Hospitalization data were obtained from the "Maintenance et exploitation de données pour l'étude de la clientèle hospitalière" (Med-Echo) database. Emergency department data (e.g., reasons for ED visits) were provided by the "Banque de données commune des urgences" (BDCU) database, while the "Système dinformation clinique et administrative des centres locaux de services communautaires" (ICLSC) database provided complementary data on psychosocial interventions and medical care provided by salaried GP in community healthcare centers. Data from these sources were merged for each patient using a unique encrypted identifier.

\section{Variables}

The dependent variables were frequency of ED use and frequency of hospitalization for $\mathrm{MH}$ reasons at 12 months following index ED visits in 2014-2015, with MH including SRD and suicidal behaviors (e.g., ideation, attempt). Emergency department use or hospitalization for physical conditions (primary or secondary diagnoses) were excluded. Main independent variables were the two groups compared: MD-CPI and MD only. Control variables included clinical, sociodemographic, and service use variables. Clinical variables involved $\mathrm{MD}, \mathrm{SRD}$, and $\mathrm{CPI}$, with severity levels $(0-3+)$ based on the Elixhauser Comorbidity Index $(44,45)$. Mental Disorders included common MD (adjustment, depressive, and anxiety disorders), serious MD (schizophrenia spectrum and other psychotic disorders, bipolar disorders), and personality disorders, while SRD included alcohol and drug use and induced disorders, intoxication, and withdrawal. Chronic physical illnesses consisted of major illness categories (e.g., hypertension, liver or valvular illnesses, coagulopathy). The four diagnoses categories involving MD and SRD were excluded from the original Elixhauser Comorbidity Index list of 31 CPI $(44,46)$. Diagnostic codes for MD, SRD, and CPI in the RAMQ were based on the International Classification of Diseases Ninth Revision (ICD-9) and those in the MED-ECHO and BDCU on the Tenth Revision (ICD-10-CA) (Table 1). These RAMQ codes for case definition have good reliability and sensitivity $(43,47,48)$.

Sociodemographic variables measured at index ED visits included age, sex, and material and social deprivation. Deprivation indices were calculated based on individual place of residence as determined by postal code and reported in the 2011 Canadian census. The Material Deprivation Index measures the ratio of population employment, average income, and number of individuals without a high school diploma for a given area; while the Social Deprivation Index calculates proportions of individuals living alone, without spouse, and single-parent families (49). Both indices are classified in quintiles, the fifth representing highest level of deprivation. In this study, material and social deprivation were regrouped and tested into three groups: least deprived (quintiles 1-2), moderately deprived (quintile 3), and most deprived (quintiles 4-5) or with no assignment, including individuals who were homeless, incarcerated, or living in institutions such as nursing homes.

Service use variables measured at 12-month follow-up were controlled for intensity, continuity, and diversity of patient care, as possible influences on ED use and hospitalization. These variables included frequency of consultations with usual GP, or usual outpatient psychiatrist; main physician provider (none, GP only, psychiatrist only, both GP, and psychiatrist); continuity of physician care; and number of psychosocial interventions in community healthcare centers. To qualify as "usual GP," at least two consultations had to have been made with a single GP, or at least two consultations with more than one GP working in the same family medicine group (50). Regarding "usual psychiatrist," patients who made only one outpatient psychiatric consultation had to have made at least two consultations with their GP, referred to as collaborative care (51). Continuity of physician care was measured with the Usual Provider Continuity Index, which described the proportion of visits to the usual GP and usual outpatient psychiatrist of total visits made (52), with a score $\geq 0.61$ as the cut-off for high continuity of care (53).

\section{Data Analyses}

Descriptive analyses were performed comparing the two main independent variables (patients with MD-CPI and MD only) and sociodemographic, clinical, and service use control variables and both dependent variables (or outcomes: ED use and hospitalization). Frequencies and percentages were calculated for all variables. As missing values were fewer than $0.5 \%$, complete case analysis was used (54). The Intraclass Correlation Coefficient (ICC) was small (0.036), indicating low correlation among patients in hospital settings making further multilevel analysis unnecessary. Intercorrelation tests were also conducted, analyzing associations between each independent and control variable; those with correlation coefficients $>0.7$ were eliminated. Negative binomial models were developed to explore differences between the two main independent variables (MD-CPI and MD 
TABLE 1 | Codes for mental disorders (MD), substance-related disorders (SRD), and chronic physical illnesses (CPI) according to the International Classification of Diseases, Ninth and Tenth revisions ${ }^{\mathrm{a}}$.

\begin{tabular}{lll}
\hline Diagnoses & $\begin{array}{l}\text { International Classification of Diseases, Ninth } \\
\text { Revision (ICD-9) }\end{array}$ & $\begin{array}{l}\text { International Classification of Diseases, Tenth } \\
\text { Revision, Canada (ICD-10-CA) }\end{array}$
\end{tabular}

\section{Mental disorders (MD) \\ Common MD}

Adjustment disorders

Depressive disorders

Anxiety disorders

\section{Serious MD}

Schizophrenia spectrum and other psychotic disorders

Bipolar disorders

\section{Personality disorders}

Substance-related disorders (SRD)

Alcohol-related disorders

Drug-related disorders

\section{Chronic physical illnesses (CPI)}

Renal failure

Cerebrovascular illnesses

Neurological illnesses

Endocrine illnesses (excluding diabetes mellitus)

Any tumor and metastatic cancer

Chronic pulmonary illnesses

Diabetes complicated and uncomplicated

Cardiovascular illnesses
309.0-309.4, 309.8, 309.9

$300.4,311.9$

300 (except 300.4)

295, 297, 298

296.0-296.6, 296.8, 296.9

301

291.0, 291.8 (alcohol withdrawal); 303.0, 303.9, 305.0 (alcohol abuse or dependence); 291.1-291.5, 291.9, 357.5, 425.5, 535.3, 571.0-571.3 (other alcohol-induced disorders); 980.0, 980.1, 980,8, 980.9 (alcohol intoxication)

292.0 (drug withdrawal); 304.0-304.9, 305.2-305.7, 305.9 (drug abuse or dependence); 292.1, 292.2, 292.8, 292.9 (other drug-induced disorders)965.0, 965.8, 967.0, 967.6, 967.8, 967.9, 969.4-969.9, 970.8, 982.0, 982.8 (drug intoxication)
403.0, 403.1, 403.9, 404.0, 404.1, 404.9, 585, 586, 588.0, V42.0, V45.1, V56

362.34, 430.x-438.x

$331.9,332.0,332.1,333.4,333.5,333.9,334-335$, $336.2,340,341,345,348.1,348.3,780.3,784.3$

240.9, 243.x, 244.x, 246.1, 246.8, 278.0, 253.6, 276.x

140.x-172.x, 174.x, 175.x, 179.x-195.x, 196.x-199.x, 200.x, 201.x, 202.x, 203.0, 238.6, 273.3

$490 x-505 . x, 506.4,508.1,508.8$

250.0-250.2, 250.3, 250.4-250.9

394-397, 424, 746.3-746.6, V42.2, V43.3, 401, 402-405, 437.2, 398.9, 402.0, 402.1, 402.9, 410, 412, 415.0, 415.1, 416, 417.0, 417.8, 417.9, 428, 426.0, 426.1, 426.5-426.7, 426.9, 427.0-427.4, 427.6-427.9, 437.2, 785.0, 996.0, V45.0, V53.3, 093, 440, 441, 443.1-443.9, 447.1, 557.1, 557.9, V43.4
F43.0-F43.2; F43.8, F43.9

F32.0-F32.8, F32.9, F33.0; F33.8, F33.9, F34.8, F34.9, F38.0, F38.1, F38.8, F39, F41.2

F40-F48, F68

F20, F21, F22, F23, F24, F25, F28, F29, F32.3; F33.3; F44.89

F30.0-F30.2, F30.8, F30.9, F31.0-F31.9

F60, F07.0, F34.0, F341, F48.8, F61

F10.1, F10.2 (alcohol abuse or dependence); F10.3, F10.4 (alcohol withdrawal); F10.5-F10.9, K70.0-K70.4, K70.9, G62.1, 142.6, K29.2, K85.2, K86.0, E24.4, G31.2, G72.1, O35.4 (other alcohol-induced disorders); F10.0, T51.0, T51.1, T51.8, T51.9 (alcohol intoxication) F11.1, F12.1, F13.1, F14.1, F15.1, F16.1, F18.1, F19.1, F11.2, F12.2, F13.2, F14.2, F15.2, F16.2, F18.2, F19.2, F55 (drug abuse or dependence);); F11.3-F11.4, F12.3, F12.4; F13.3-F13.4, F14.3-F14.4, F15.3-F15.4, F16.3-F16.4, F18.3-F18.4, F19.4-F19.4 (drug withdrawal); F11.5-F11.9, F12.5-F12.9; F13.5-F13.9, F14.5-F14.9, F15.5-F15.9, F16.5-F16.9, F18.5-F18.9, F19.5-F19.9 (other drug-induced disorders; F11.0, F12.0, F13.0, F14.0, F15.0, F16.0, F18.0, F19.0, T40.0-T40.9, T42.3, T42.4, T42.6, T42.7, T43.5, T43.6, T43.8, T43.9, T50.9, T52.8, T52.9 (drug intoxication)

I12.0, I13.1, N18.x, N19.x, N25.0, Z49.x, Z94.0, Z99.2

G45.x, G46.x, 160.x-169.x

G10.x-G12.x, G13.x, G20.x, G21.x-G22.x, G25.4, G25.5, G31.8, G31.9, G32.x, G35.x, G36.x, G37.x, G40.x, G41.x, G93.1, G93.4, R47.0, R56.x

E66.x, E00.x, E01.x, E02.x, E03.x, E89.0, E22.2, E86.x, E87.x

C00.x-C26.x, C30.x-C34.x, C37.x-C41.x, C43.x, C45.x-C58.x, C60.x-C76.x, C77.x-C79.x, C80.x C81.x-C85.x, C88.x, C90.0, C90.2, C96.x

I27.8, I27.9, J40.x-J47.x, J60.x-J64.x, J65.x, J66.x, J67.x, J68.4, J70.1, J70.3

E10.2-E10.8, E11.2-E11.8, E13.2-E13.8, E14.2-E14.8, E10.0, E10.1, E10.9, E11.0, E11.1, E11.9, E13.0, E13.1, E13.9, E14.0, E14.1, E14.9

105.x-108.x, 109.1, 109.8, 110.x, I11.x-113.x, I15.x, 167.4, I09.9, I11.0, I13.0, I13.2, I21.x, I22.x, I25.2, I25.5, I26.x, I27.x, I28.0, I28.8, I28.9, I34.x-I39.x, |42.0, 142.5|42.9, 143.x, 150.x, P29.0, 144.1-144.3, 145.6, 145.9, 147.x-149.x, Q23.0-Q23.3, Q23.8, Q23.9 R00.0, R00.1, R00.8, T82.1, Z45.0, Z95.0, Z95.2, Z95.3, Z95.4, A52.0, I70.x, 171.x,I72.x, I73.0, 173.1, I73.8, 173.9, I77.1, 179.0, K55.1, K55.8, K55.9, Z95.8, Z95.9 
TABLE 1 | Continued

\begin{tabular}{|c|c|c|}
\hline Diagnoses & $\begin{array}{l}\text { International Classification of Diseases, Ninth } \\
\text { Revision (ICD-9) }\end{array}$ & $\begin{array}{l}\text { International Classification of Diseases, Tenth } \\
\text { Revision, Canada (ICD-10-CA) }\end{array}$ \\
\hline $\begin{array}{l}\text { Other CPI categories (blood loss anemia, ulcer, } \\
\text { liver illnesses, AIDS/HIV, rheumatoid } \\
\text { arthritis/collagen vascular illnesses, } \\
\text { coagulopathy, weight loss, paralysis, deficiency } \\
\text { anemia) }\end{array}$ & $\begin{array}{l}\text { 280.0, 280.90, 286.x, 287.1, 287.3-287.5 531.7, } \\
\text { 531.9, 532.7, 532.9, 533.7, 533.9, 534.7, 534.9, } \\
\text { 070.22, 070.23, 070.2, 070.32, 070.33, 070.3, } \\
\text { 070.44, 070.4, 070.54, 070.5, 456.0-456.2, 572.3, } \\
\text { 572.8, 573.3, 573.4, 573.9, V42.7, 042.x-044.x, } \\
\text { 136.1, 446.x, 701.0, 710.0-710.4, 710.5, 710.8, } \\
\text { 710.9, 711.2, 714.x, 719.3, 720.x, 725.x, 728.5, } \\
\text { 728.8, 729.30, 260.x-263.x, 783.2, 799.4, 334.1, } \\
\text { 342.x, 343.x, 344.0, 344.1, 344.2, 344.3, 344.4, } \\
\text { 344.5, 344.6, 344.8, 344.9, 280.1-289.0, } \\
\text { 280.91-280.0 281.x, 285.9, }\end{array}$ & $\begin{array}{l}\text { B20.x-B24.x, D50.0, D65-D68.x, D69.1, D69.3-D69.6 } \\
\text { K25.7, K25.9, K26.7, K26.9, K27.7, K27.9, K28.7, } \\
\text { K28.9, B18.x, I85.x, I86.4, I98.2, K71.1, K71.3-K71.5, } \\
\text { K71.6, K71.7, K72.1, K72.9, K73.x, K74.x, K75.4, } \\
\text { K76.0, K76.1, K76.3, K76.4, K76.5, K76.6, K76.8, } \\
\text { K76.9, Z94.4, L90.0, L94.0, L94.1,L94.3, M05.x, M06.x, } \\
\text { M08.x, M12.0, M12.3, M30.x, M31.x, M32.x-M35.x, } \\
\text { M45.x, M46.0, M46.1, M46.8, M46.9x, G04.1, G11.4, } \\
\text { G80.x, G81.x, G82.x, G83.x, E40.x-E46.x, R63.4, } \\
\text { R64.x, D50.1, D50.8, D50.9, D51.x-D53.x, D63.x, D64.9 }\end{array}$ \\
\hline
\end{tabular}

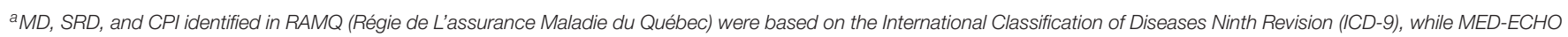

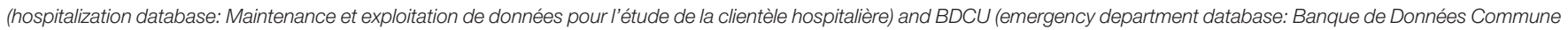
des Urgences) were based on the ICD Tenth Canadian Revision (ICD-10-CA).

only) on the outcomes, frequency of ED use, and frequency of hospitalization for $\mathrm{MH}$ reasons at 12-month follow-up to the 2014-2015 index visit, controlling for key clinical, sociodemographic, and service use variables. Negative binomial analysis showed better goodness-of-fit than results for the zeroinflated models. Based on the Akaike's Information Criterion (AIC) and Bayesian Information Criterion (BIC), models with the smallest AIC or BIC were chosen as the final multivariate models. Interactions between sex and patient group were not significant. Data analysis was performed using STATA 17.0 software (54).

\section{RESULTS}

At 12-month follow-up, $10 \%$ of the cohort (95\% CI: 9-11) had made no ED visits, 51\% (95\% CI: 48-52) only one, and 39\% (95\% CI: 37-40) two or more visits for $\mathrm{MH}$ reasons, with a mean of 1.82 visits (SD: 2.08; range: 0-26; median: 1; IQR: 1 ), while $77 \%$ were not hospitalized (mean: 0.29; SD: 0.62; range: 0-9; median: 0; IQR:1). Of the 2,677 patients, 52\% (95\% CI: 50-54) had common MD, 19\% (95\% CI: 17-20) serious MD, 12\% (95\% CI: 11-13) personality disorders, and 21\% (95\% CI: 20-23) SRD (Table 2). Regarding CPI, cardiovascular illnesses (35\%; 95\% CI: 33-37) were most prevalent, followed by chronic pulmonary illnesses, and complicated or uncomplicated diabetes (16\% each; $95 \%$ IC: 14-18) (Figure 1). However, CPI severity index were low, with $89 \%$ (95\% CI: 87-90) having a score of 0. For this cohort, $37 \%$ (95\% CI: 35-39) of patients were 25-44 years old and 52\% (95\% CI: 50-54) were men. Ratings of 4 and 5 or not assigned, in the Material and Social Deprivation Indices were 45\% (95\% CI: 4347) for material deprivation and 62\% (95\% CI: 60-64) for social deprivation. Following index ED visits, 51\% (95\% CI: 49-52) and $68 \%$ (95\% CI: 67-70) reported no consultation with their usual GP or psychiatrist; 37\% (CI: 35-39) of the sample had no main physician provider (either GP or psychiatrist), while GP were assigned as the main providers for 31\% (95\% CI: 29-33\%). For the entire sample, $41 \%$ (95\% CI: 39-42) received high scores for continuity of physician care, and 30\% (95\% CI: 28-33) for psychosocial interventions in community healthcare centers.
Table 3 shows comparative results for MD-CPI and MD only on frequency of ED use at 12-month follow-up, controlling for clinical, sociodemographic, and service use variables. Risk of ED use among patients with MD-CPI increased 17\% (IRR = 1.17 ; $95 \% \mathrm{CI}=1.05-1.30$ ) compared to those with MD only. Substance-related disorders increased the risk by 39\% (IRR = 1.39 ; $95 \% \mathrm{CI}=1.23-1.58$ ), while severity scores for chronic illnesses ranged from 1 to 3 , increasing risk by $39 \%$ (IRR $=1.39$; $95 \% \mathrm{CI}=1.17-1.65)$ for patients with a severity score of 1 and by $40 \%$ (IRR $=1.40 ; 95 \% \mathrm{CI}=1.17-1.67)$ for those scoring 3. Patients 65 years + had $25 \%(\mathrm{IRR}=0.75 ; 95 \% \mathrm{CI}=0.64-$ 0.84) less risk of ED use vs. those 12-24 years old. Emergency department use increased to $25 \%$ (IRR $=1.25$; 95\% CI $=1.12-$ 1.39) among patients having 1-3 consultations with their usual psychiatrist or 1-3 psychosocial interventions in community healthcare centers (IRR $=1.25$; 95\% CI $=1.14-1.37$ ); while ED use augmented of $31 \%$ among patients having $4+$ consultations $(\mathrm{IRR}=1.31 ; 95 \% \mathrm{CI}=1.18-1.46)$ with their usual psychiatrist and of $27 \%($ IRR $=1.27 ; 95 \% \mathrm{CI}=1.14-1.40)$ among those having $4+$ psychosocial interventions in community healthcare centers. High continuity of physician care decreased the risk of $t$ ED use by $23 \%(I R R=0.77 ; 95 \% \mathrm{CI}=0.69-0.86)$.

Table 4 presents results for MD-CPI compared with MD only on hospitalization at 12-month follow-up, controlling for the same variables as above. Patients with MD-CPI had 59\% (IRR $=1.59 ; 95 \% \mathrm{CI}=1.30-1.94)$ greater risk of hospitalization than those with MD only. Serious MD increased the risk of hospitalization by $62 \%(\mathrm{IRR}=1.62$; $95 \% \mathrm{CI}=1.36-1.92)$. Risk of hospitalization for patients with common MD decreased by $37 \%$ (IRR $=0.63 ; 95 \% \mathrm{CI}=0.53-0.74)$ and for patients with personality disorders by $32 \%$ (IRR $=0.68 ; 95 \% \mathrm{CI}=0.51-0.89$ ). Compared with patients 12-24 years old, those 45-64 years were $26 \%(\mathrm{IRR}=1.26 ; 95 \% \mathrm{CI}=1.01-1.56)$ and those $65+$ were $88 \%$ $(\mathrm{IRR}=1.88 ; 95 \% \mathrm{CI}=1.41-2.49)$ more likely to be hospitalized. Men were at $17 \%(\operatorname{IRR}=1.17 ; 95 \% \mathrm{CI}=1.01-1.36)$ greater risk for hospitalization than women. Patients who received 13 outpatient psychiatric consultations were 1.89 times $($ IRR $=$ 2.89; $95 \% \mathrm{CI}=2.29-3.65)$ more likely to be hospitalized, while the likelihood for those with $4+$ consultations increased 3.25 
TABLE 2 | Characteristics of patients at onset of a mental disorder (MD) including substance-related disorders (SRD) who used emergency departments (ED) in the index year 2014-15.

\begin{tabular}{|c|c|c|c|c|c|c|}
\hline \multirow[t]{2}{*}{ Characteristics } & \multicolumn{2}{|c|}{ Study sample patients } & \multicolumn{2}{|c|}{$\begin{array}{l}\text { MD and chronic physical } \\
\text { illnesses (CPI) group }\end{array}$} & \multicolumn{2}{|c|}{ MD group only } \\
\hline & $N$ & $\%(95 \% \mathrm{Cl})$ & $N$ & $\%(95 \% \mathrm{Cl})$ & $N$ & $\%(95 \% \mathrm{Cl})$ \\
\hline Overall & 2,677 & 100 & 678 & & 1,999 & 100 \\
\hline \multicolumn{7}{|c|}{ Clinical variables (2012-13 to index ED visit) } \\
\hline \multicolumn{7}{|l|}{ Mental disorders (MD) ${ }^{\mathrm{a}}$} \\
\hline Common MD & 1,399 & $52.3(50.3-54.1)$ & 335 & $49.4(45.6-53.1)$ & 1,064 & $53.2(51.0-55.4)$ \\
\hline Serious MD & 499 & $18.6(17.2-20.1)$ & 148 & $21.8(18.8-25.1)$ & 351 & $17.6(15.9-19.2)$ \\
\hline Personality disorders & 324 & $12.1(10.9-13.3)$ & 67 & $9.9(7.8-12.3)$ & 257 & $12.9(11.4-14.3)$ \\
\hline Substance-related disorders (SRD) & 568 & $21.2(19.7-22.8)$ & 144 & $21.2(18.3-24.4)$ & 424 & $21.2(19.4-23.0)$ \\
\hline \multicolumn{7}{|c|}{$\begin{array}{l}\text { Elixhauser Comorbidity Index (severity of chronic } \\
\text { physical illnesses - CPI) }\end{array}$} \\
\hline 0 & 2,374 & $88.6(87.4-89.8)$ & 375 & $55.3(51.5-59.0)$ & 1,999 & 100 \\
\hline 1 & 119 & $4.4(3.7-5.2)$ & 119 & $17.6(14.8-20.6)$ & 0 & 0 \\
\hline 2 & 75 & $2.8(2.2-3.4)$ & 75 & $11.1(8.9-13.6)$ & 0 & 0 \\
\hline $3+$ & 109 & $4.0(3.3-4.8)$ & 109 & $16.1(13.4-19.0)$ & 0 & 0 \\
\hline \multicolumn{7}{|l|}{ Sociodemographic variables (2014-15) } \\
\hline \multicolumn{7}{|l|}{ Age } \\
\hline $12-24$ years & 767 & $28.7(26.9-30.3)$ & 69 & $10.2(8.1-12.6)$ & 698 & $34.9(32.8-37.0)$ \\
\hline 25-44 years & 995 & $37.2(35.3-39.0)$ & 173 & $25.5(22.3-28.9)$ & 822 & $41.1(38.9-43.2)$ \\
\hline $45-64$ years & 702 & $26.1(24.5-27.9)$ & 273 & $40.3(36.6-44.0)$ & 429 & $21.5(19.7-23.3)$ \\
\hline $65+$ years & 213 & $7.9(6.9-9.0)$ & 163 & $24.0(20.9-27.4)$ & 50 & $2.5(1.9-3.2)$ \\
\hline \multicolumn{7}{|l|}{ Sex } \\
\hline Men & 1,395 & $52.1(46.0-49.7)$ & 330 & $48.7(44.9-52.4)$ & 1,065 & $53.3(51.0-55.4)$ \\
\hline Women & 1,282 & $47.8(50.2-54.0)$ & 348 & $51.3(47.5-55.0)$ & 934 & $46.7(44.5-48.9)$ \\
\hline \multicolumn{7}{|l|}{ Material Deprivation Index } \\
\hline 1-2: least deprived & 984 & $36.8(34.9-38.6)$ & 229 & $33.8(30.3-37.4)$ & 755 & $37.8(35.6-39.9)$ \\
\hline 3 & 481 & $18.0(16.5-19.4)$ & 121 & $17.8(15.1-20.9)$ & 360 & $18.0(16.3-19.7)$ \\
\hline $4-5$ most deprived or not assigned ${ }^{c}$ & 1,212 & $45.2(43.3-47.1)$ & 328 & $48.4(44.6-52.1)$ & 884 & $44.2(42.0-46.4)$ \\
\hline \multicolumn{7}{|l|}{ Social Deprivation Index } \\
\hline 1-2: least deprived & 633 & $23.3(22.0-25.2)$ & 151 & $22.2(19.2-25.5)$ & 482 & $24.1(22.2-26.0)$ \\
\hline 3 & 387 & $14.5(13.1-15.8)$ & 92 & $13.6(11.1-16.3)$ & 295 & $14.8(13.2-16.3)$ \\
\hline 4-5: most deprived or not assigned ${ }^{C}$ & 1,657 & $61.8(60.0-63.7)$ & 435 & $64.2(60.4-67.6)$ & 1,222 & $61.1(58.9-63.2)$ \\
\hline \multicolumn{7}{|c|}{$\begin{array}{l}\text { Service use variables (12-month follow-up to index ED } \\
\text { visit in 2014-15) }\end{array}$} \\
\hline \multicolumn{7}{|c|}{$\begin{array}{l}\text { Frequency of consultations with usual general } \\
\text { practitioner (GP) }\end{array}$} \\
\hline 0 consultation & 1,354 & $50.6(48.6-52.4)$ & 257 & $37.9(34.3-41.6)$ & 1,097 & $54.9(52.6-57.0)$ \\
\hline $1-3$ consultations & 622 & $23.2(21.6-24.8)$ & 181 & $26.7(23.4-30.1)$ & 441 & $22.1(20.2-23.9)$ \\
\hline $4+$ consultations & 701 & $26.2(24.5-27.8)$ & 240 & $35.4(31.8-39.0)$ & 461 & $23.0(21.2-24.9)$ \\
\hline \multicolumn{7}{|c|}{$\begin{array}{l}\text { Frequency of consultations with usual outpatient } \\
\text { psychiatrist }^{\mathrm{e}}\end{array}$} \\
\hline 0 consultation & 1,830 & $68.4(66.5-70.0)$ & 452 & $66.7(63.0-70.1)$ & 1,378 & $68.9(66.8-70.9)$ \\
\hline $1-3$ consultations & 385 & $14.4(13.1-15.7)$ & 91 & $13.4(11.0-16.2)$ & 294 & $14.7(13.2-16.3)$ \\
\hline $4+$ consultations & 462 & $17.3(15.8-18.7)$ & 135 & $19.9(17.0-23.0)$ & 327 & $16.4(14.7-18.0)$ \\
\hline \multicolumn{7}{|l|}{ Main physician provider ${ }^{\dagger}$} \\
\hline GP only & 834 & $31.1(29.4-32.9)$ & 275 & $40.6(36.9-44.3)$ & 559 & $28.0(26.0-29.9)$ \\
\hline Psychiatrist only & 347 & $13.0(11.7-14.2)$ & 66 & $9.7(7.7-12.2)$ & 281 & $14.0(12.6-15.6)$ \\
\hline Both GP and psychiatrist & 500 & $18.7(17.2-20.1)$ & 160 & $23.6(20.5-26.9)$ & 340 & $17.0(15.4-15.6)$ \\
\hline None (neither GP nor psychiatrist) & 996 & $37.2(35.3-39.0)$ & 177 & $26.1(22.9-29.5)$ & 819 & $41.0(38.8-43.1)$ \\
\hline
\end{tabular}


TABLE 2 | Continued

\begin{tabular}{|c|c|c|c|c|c|c|}
\hline \multirow[t]{2}{*}{ Characteristics } & \multicolumn{2}{|c|}{ Study sample patients } & \multicolumn{2}{|c|}{$\begin{array}{l}\text { MD and chronic physical } \\
\text { illnesses (CPI) group }\end{array}$} & \multicolumn{2}{|c|}{ MD group only } \\
\hline & $N$ & $\%(95 \% \mathrm{Cl})$ & $N$ & $\%(95 \% \mathrm{Cl})$ & $N$ & $\%(95 \% \mathrm{Cl})$ \\
\hline \multicolumn{7}{|c|}{$\begin{array}{l}\text { Usual Provider Continuity Index integrating both GP and } \\
\text { psychiatrist (continuity of physician care) }\end{array}$} \\
\hline$\geq 0.61$ (high continuity of physician care) & 1,085 & $40.5(38.6-42.4)$ & 226 & $33.3(29.8-36.9)$ & 859 & $43.0(40.8-45.1)$ \\
\hline 0 intervention & 1,869 & $69.8(68.0-71.5)$ & 507 & $74.7(71.3-77.9)$ & 1,362 & $68.1(66.0-70.1)$ \\
\hline $1-3$ interventions & 463 & $17.3(15.9-18.7)$ & 92 & $13.6(11.1-16.3)$ & 371 & 18.6 (16.9-20.3) \\
\hline $4+$ interventions & 345 & $12.9(11.6-14.2)$ & 79 & $11.6(9.4-14.2)$ & 266 & $13.3(11.8-14.8)$ \\
\hline
\end{tabular}

Cl, confidence interval.

a Patients may have made more than one ED visit for MD in index year 2014-15, so total percentage may exceed $100 \%$.

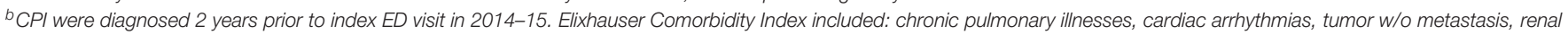

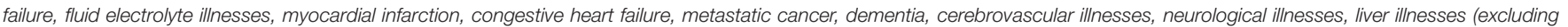

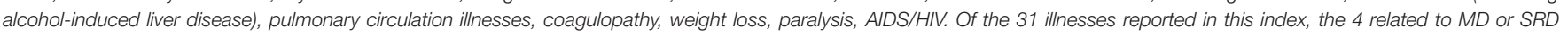
categories were excluded. This index was originally developed for mortality, and not for ED use or hospitalization, which may have impacted findings for this study.

${ }^{c}$ Missing address or living in an area where index assignment is not available. An index cannot usually be assigned to residents of nursing homes or homeless individuals.

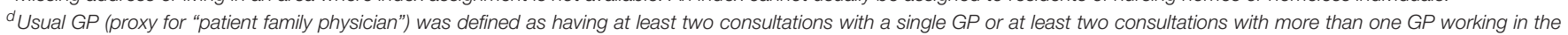
same family medicine group.

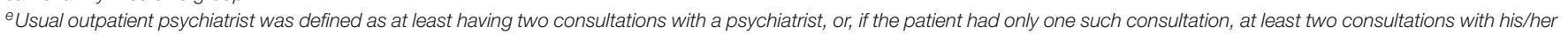
GP, referred to as collaborative care.

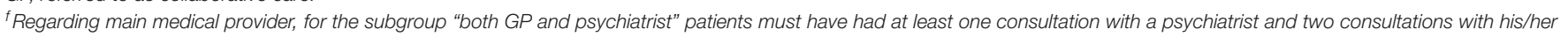
GP in outpatient care.

gUsual provider continuity index describes the proportion of visits to the usual GP and usual outpatient psychiatrist of the total GP and outpatient psychiatrist visits.

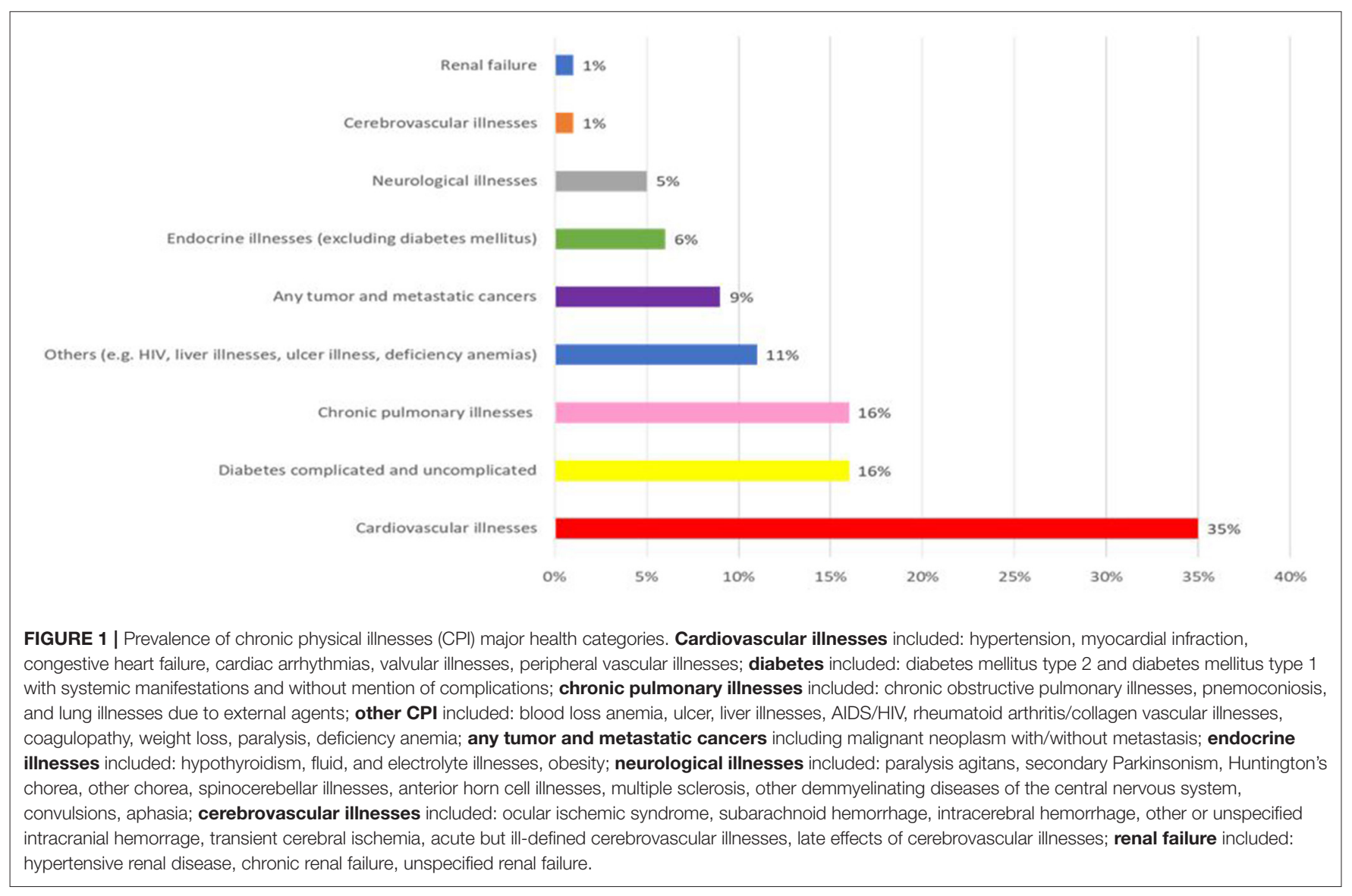


TABLE 3 | Negative binomial model comparing groups of patients diagnosed at onset of mental disorders (MD) including substance-related disorders (SRD) with or without chronic physical illnesses (CPI) on frequency of emergency department (ED) visits for mental health reasons at 12-month follow-up to index ED visit in 2014-15.

\begin{tabular}{|c|c|c|c|}
\hline & IRR $(95 \% \mathrm{Cl})$ & Adjusted IRR (95\% Cl)* & $P$-value for adjusted IRR \\
\hline \multicolumn{4}{|l|}{ Clinical variables (2012-13 to index ED visit) } \\
\hline Patient group with MD and CPI (ref.: patient group with MD only) & $1.23(1.17-1.37)$ & $1.17(1.05-1.30)$ & 0.004 \\
\hline \multicolumn{4}{|l|}{ MD at 2014-15 index ED visit (ref.: no MD) } \\
\hline Common MD & $0.98(0.91-1.05)$ & $0.98(0.91-1.05)$ & 0.65 \\
\hline Serious MD & $0.99(0.90-1.08)$ & $0.94(0.85-1.03)$ & 0.223 \\
\hline SRD vs. no SRD & $1.36(1.20-1.54)$ & $1.39(1.23-1.58)$ & $<0.001$ \\
\hline \multicolumn{4}{|l|}{ Elixhauser Comorbidity Index (severity of chronic physical illnesses) ${ }^{a}$} \\
\hline 1 vs. 0 & $1.49(1.28-1.74)$ & $1.39(1.17-1.65)$ & $<0.001$ \\
\hline 2 vs. 0 & $1.44(1.19-1.74)$ & $1.37(1.11-1.68)$ & 0.003 \\
\hline $3+$ vs. 0 & $1.44(1.23-1.69)$ & $1.40(1.17-1.67)$ & $<0.001$ \\
\hline \multicolumn{4}{|l|}{ Sociodemographic variables (2014-15) } \\
\hline \multicolumn{4}{|l|}{ Age } \\
\hline $25-44$ vs. $12-24$ years & $0.96(0.88-1.04)$ & $0.93(0.85-1.01)$ & 0.111 \\
\hline $45-64$ vs. $12-24$ years & $1.03(0.93-1.13)$ & $0.93(0.85-1.03)$ & 0.213 \\
\hline $65+$ vs. $12-24$ years & $0.87(0.75-1.01)$ & $0.75(0.64-0.88)$ & $<0.001$ \\
\hline \multicolumn{4}{|l|}{ Sex (ref.: women) } \\
\hline Men & $0.99(0.92-1.06)$ & $1.01(0.95-1.09)$ & 0.591 \\
\hline \multicolumn{4}{|l|}{ Service use variables (12-month follow-up to index ED visit in 2014-15) } \\
\hline \multicolumn{4}{|l|}{ Frequency of consultations with usual general practitioner $(\mathrm{GP})^{\mathrm{b}}$} \\
\hline $1-3$ consultations vs. none & $0.98(0.90-1.07)$ & $1.10(0.98-1.22)$ & 0.086 \\
\hline $4+$ consultations vs. none & $0.99(0.91-1.08)$ & $1.10(0.99-1.24)$ & 0.069 \\
\hline \multicolumn{4}{|l|}{ Frequency of consultations with usual outpatient psychiatrist ${ }^{\mathrm{C}}$} \\
\hline $1-3$ consultations vs. none & $1.17(1.06-1.30)$ & $1.25(1.12-1.39)$ & $<0.001$ \\
\hline $4+$ consultations vs. none & $1.18(1.07-1.29)$ & $1.31(1.18-1.46)$ & $<0.001$ \\
\hline \multicolumn{4}{|c|}{ Frequency of psychosocial interventions in community healthcare centers (excluding GP interventions) } \\
\hline $1-3$ consultations vs. none & $1.22(1.12-1.33)$ & $1.25(1.14-1.37)$ & $<0.001$ \\
\hline $4+$ consultations vs. none & $1.34(1.24-1.46)$ & $1.27(1.14-1.40)$ & $<0.001$ \\
\hline \multicolumn{4}{|c|}{ High Usual Provider Continuity Index integrating GP and psychiatrist (continuity of physician care) ${ }^{d}$} \\
\hline$\geq 0.61$ (high continuity of physician care) vs. < 0.61 (low continuity of physician care) & $0.94(0.87-1.01)$ & $0.77(0.69-0.86)$ & $<0.001$ \\
\hline
\end{tabular}

Confidence interval (Cl); incidence rate ratio (IRR).

${ }^{*}$ Adjusted IRR for all variables.

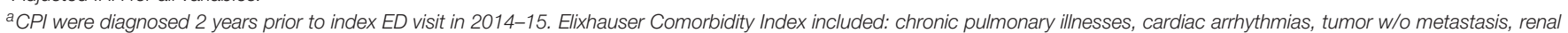

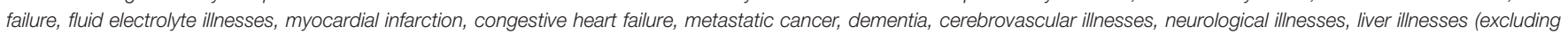

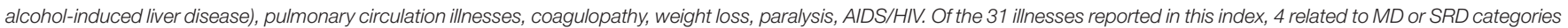
were excluded. This index was originally developed for mortality, not for ED use or hospitalization, which may have affected findings for this study.

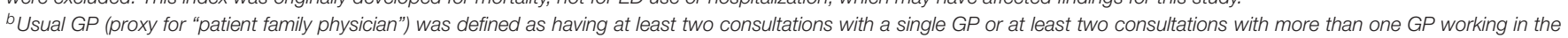
same family medicine group.

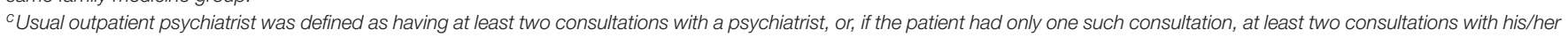
GP, referred to as collaborative care.

${ }^{d}$ High usual Provider Continuity Index describes the proportion of visits to the usual GP and usual outpatient psychiatrist of total GP and outpatient psychiatrist visits.

times $(\mathrm{IRR}=4.25 ; 95 \% \mathrm{CI}=3.47-5.22)$. By contrast, risk of hospitalization decreased to $36 \%$ (IRR $=0.64$; $95 \% \mathrm{CI}=0.52-$ 0.79 ) for those receiving high continuity of physician care, and to $29 \%(\mathrm{IRR}=0.71 ; 95 \% \mathrm{CI}=0.57-0.88)$ after $4+$ consultations with the usual patient GP.

\section{DISCUSSION}

This study was original in measuring frequencies of both ED use and hospitalization for $\mathrm{MH}$ reasons, comparing patients with MD-CPI and those with MD only, and controlling for multiple clinical, sociodemographic, and service use variables. Overall, we found that one patient in four had MD-CPI. As patients recruited from ED tend to be affected with serious or complex $\mathrm{MD}$, and most in this sample also experienced material or social deprivation, high prevalence of MD-CPI was expected. As well, most patients had used ED at least once by 12-month follow-up, if not several times, while nearly one fourth were hospitalized.

Patients with MD-CPI were more likely to make frequent use of $\mathrm{ED}$ and to be hospitalized for $\mathrm{MH}$ reasons than those with MD only during the 12-month follow-up period, confirming hypothesis one. Previous studies found that frequent psychiatric ED users were often affected by co-occurring CPI $(7,14)$. A recent systematic review also identified higher risk of psychiatric hospitalization among patients with MD-CPI (17). The present study was however one of the first to estimate levels of risk 
TABLE 4 | Negative binomial model comparing groups of patients at onset of mental disorders (MD) including substance-related disorders (SRD) with or without chronic physical illnesses (CPI) on frequency of hospitalization for mental health reasons at 12-month follow-up to index ED visit in 2014-15.

\begin{tabular}{|c|c|c|c|}
\hline & IRR & Adjusted IRR $(95 \% \mathrm{Cl})^{\star}$ & $P$-value for adjusted IRR \\
\hline \multicolumn{4}{|l|}{ Clinical variables (2012-13 to index ED visit) } \\
\hline Patient group with MD and CPI (ref.: patient group with MD only) & $2.04(1.73-2.40)$ & $1.59(1.30-1.94)$ & $<0.001$ \\
\hline \multicolumn{4}{|l|}{ MD at 2014-15 index ED visit (ref.: no MD) } \\
\hline Common MD & $0.47(0.40-0.56)$ & $0.63(0.53-0.74)$ & $<0.001$ \\
\hline Serious MD & $3.04(2.59-3.57)$ & $1.62(1.36-1.92)$ & $<0.001$ \\
\hline Personality disorders & $0.64(0.48-0.85)$ & $0.68(0.51-0.89)$ & $<0.005$ \\
\hline SRD vs. no SRD & $1.07(0.80-1.45)$ & $1.21(0.92-1.59)$ & 0.169 \\
\hline \multicolumn{4}{|l|}{ Elixhauser Comorbidity Index (severity of chronic physical illnesses) ${ }^{a}$} \\
\hline 1 vs. 0 & $1.40(0.98-1.99)$ & $1.01(0.72-1.44)$ & 0.911 \\
\hline 2 vs. 0 & $1.87(1.26-2.79)$ & $1.06(0.73-1.45)$ & 0.738 \\
\hline $3+$ vs. 0 & $1.97(1.42-2.73)$ & $1.28(0.92-1.75)$ & 0.133 \\
\hline \multicolumn{4}{|l|}{ Sociodemographic variables (2014-15) } \\
\hline \multicolumn{4}{|l|}{ Age } \\
\hline $25-44$ vs. $12-24$ years & $1.13(0.92-1.40)$ & $1.16(0.95-1.41)$ & 0.139 \\
\hline $45-64$ vs. $12-24$ years & $1.41(1.13-1.75)$ & $1.26(1.01-1.56)$ & 0.033 \\
\hline $65+$ vs. $12-24$ years & $2.31(1.76-3.05)$ & $1.88(1.41-2.49)$ & $<0.001$ \\
\hline \multicolumn{4}{|l|}{ Sex } \\
\hline Men vs. women & $1.17(1.00-1.38)$ & $1.17(1.01-1.36)$ & 0.036 \\
\hline \multicolumn{4}{|l|}{ Service use variables (12-month follow-up to index ED visit in 2014-15) } \\
\hline \multicolumn{4}{|l|}{ Frequency of consultations with usual general practitioner $(\mathrm{GP})^{\mathrm{b}}$} \\
\hline $1-3$ consultations vs. none & $0.84(0.69-1.03)$ & $0.84(0.68-1.02)$ & 0.100 \\
\hline $4+$ consultations vs. none & $0.65(0.53-0.80)$ & $0.71(0.57-0.88)$ & 0.002 \\
\hline \multicolumn{4}{|l|}{ Frequency of consultations with usual outpatient psychiatrist ${ }^{c}$} \\
\hline $1-3$ consultations vs. none & $2.15(1.74-2.65)$ & $2.89(2.29-3.65)$ & $<0.001$ \\
\hline $4+$ consultations vs. none & $4.03(3.40-4.78)$ & $4.25(3.47-5.22)$ & $<0.001$ \\
\hline \multicolumn{4}{|l|}{ High Usual Provider Continuity Index integrating GP and psychiatrist (continuity of physician care) ${ }^{d}$} \\
\hline$\geq 0.61$ (high continuity of physician care) vs. $<0.61$ (low continuity of physician care) & $1.13(0.96-1.33)$ & $0.64(0.52-0.79)$ & $<0.001$ \\
\hline
\end{tabular}

Confidence interval (Cl); incidence rate ratio (IRR).

${ }^{\star}$ Adjusted IRR for all variables.

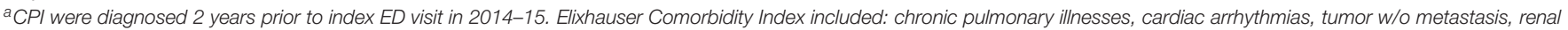

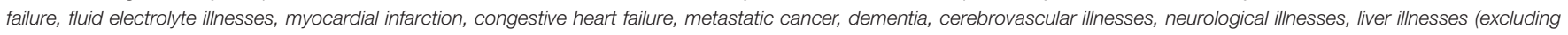

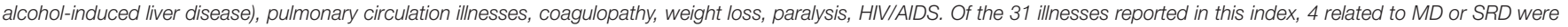
excluded. This index was originally developed for mortality, not for ED use or hospitalization, which may have affected findings for this study.

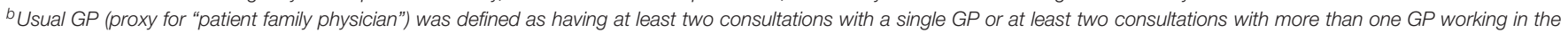
same family medicine group.

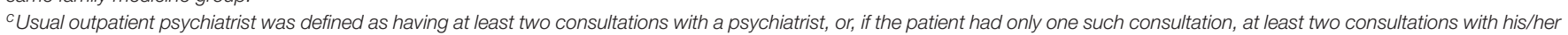
GP, referred to as collaborative care.

${ }^{d}$ High usual Provider Continuity Index describes the proportion of visits to the usual GP and usual outpatient psychiatrist of total GP and outpatient psychiatrist visits.

for ED use and hospitalization for $\mathrm{MH}$ reasons among patients with MD-CPI, compared to patients with MD only. Overall, the increased risk for ED use was significant, but modest (15\%), while hospitalization risk was substantially elevated at $63 \%$.

The findings also partially confirm the second hypothesis that having more serious medical conditions, including SRD or severe CPI, would increase ED use, and that serious MD would increase hospitalization rates. Patients with SRD are known to be high ED users $(55,56)$ and to use few outpatient services in addition to low treatment compliance or motivation $(57,58)$. Severity of CPI was found to increase the risks of ED use and hospitalization for medical reasons (17, 24, 33). Studies have also identified CPI as key determinants of high $\mathrm{ED}$ use for $\mathrm{MH}$ reasons, and serious $\mathrm{MD}$ an added impetus for hospitalization $(59,60)$. Other studies reported that patients with MD-CPI were less likely than others to receive care for CPI from their GP $(61,62)$, suggesting that they may have used psychiatric ED for medical issues. Studies on determinants of hospitalization for medical reasons found a relationship between personality disorders and low hospitalization (59, 63). This may be partially explained by negative attitudes among hospital staff and stigmatization experienced by patients with personality disorders, and particularly borderline personality disorder, who are viewed as difficult patients, often manipulative or violent $(64,65)$. Hospitalization is usually considered inappropriate for these patients, except in cases of acute crisis requiring shortstay admission (64). Treatments other than hospitalization are recommended for patients with personality disorders, including 
outpatient psychiatric treatment (66) or psychosocial treatments $(67,68)$. The finding that risk of hospitalization decreased among patients with common MD is explained by research showing the effectiveness of primary care treatment for adjustment, anxiety and depressive disorders $(69,70)$, and that these conditions require less frequent hospitalization than serious MD.

Regarding the third hypothesis related to quality of services, only high continuity of physician care protected against both ED use and hospitalization, while intensive care from the usual GP prevented hospitalization. High continuity of physician care is a key recovery indicator, particularly among patients with MD, CPI, or co-occurring diagnoses $(71,72)$. Previous studies have demonstrated that higher continuity of care is associated with less frequent ED use $(8,73)$ and hospitalization $(74)$. Receiving intensive care from GP (4+ consultations yearly) after onset of MD, another continuity of care measure and key indicator for quality of care and patient recovery $(75,76)$, was found to decreased frequency of hospitalization. It is possible that access to GP for consultations by patients was not sufficiently rapid that their use of ED for MH problems could be avoided or reduced. Patients with MD or both MD-CPI often face crisis situations involving psychological distress or suicidal behaviors, which may also exacerbate co-occurring problems. As such, close follow-up by GP may have helped patients avoid decompensation, while supporting their adherence to treatment and motivation to seek care, as well as protecting them against hospitalization. Some $50 \%$ of patients in the sample also reported making no GP consultations and 4 of 10 patients had no medical provider, pointing to important unresolved issues involving access to physician care for this population.

Surprisingly, the study revealed that receiving more intensive outpatient care from psychiatrists increased the risks of both frequent $\mathrm{ED}$ use and hospitalization. This result may point to a certain lack of appropriateness or diversity in outpatient psychiatric treatments commensurate with the needs of patients affected by very complex and serious conditions requiring highly specialized care. Previous research has shown similar results $(14,59)$. Community healthcare centers also offered psychosocial interventions to more vulnerable individuals who which perhaps had led to these patient higher risks of frequent ED use. These organizations also provided more treatments for individuals with common $\mathrm{MH}$ problems, which may explain the lack of association with risk of hospitalization.

Among sociodemographic variables, age was associated with both ED use and hospitalization, while sex was associated with hospitalization only. Compared with patients in the 1224 age group, those $65+$ were less likely to use $\mathrm{ED}$ for $\mathrm{MH}$ reasons, possibly because MD are often underdiagnosed or undertreated among older patients, whereas CPI are more commonly addressed $(77,78)$. Yet patients $65+$, as well as those in the 45-64 age group, were at relatively greater risk for hospitalization. Sources of care other than hospitalization are usually preferred for younger patients, due to perceived stigma $(79,80)$. Moreover, the finding that men had an elevated risk of hospitalization may be explained by research suggesting that men generally use services as a last resort, once their $\mathrm{MH}$ problems become quite serious (81). Men also use primary care less than women (82).
This study had several limitations. First, Quebec medical administrative databases were developed primarily for financial purposes, and as such the results represent only a proxy for patient needs. Second, key data including ethnicity, medication compliance, side-effects of anti-psychotic medications, and use of psychosocial hospital teams, community $\mathrm{MH}$ organizations or psychologists in private practice that may have potentially impacted frequency of ED use and hospitalization were not available from these study databases. Third, use of the ICD9 codification and not ICD-10 from the RAMQ database, and use of the Elixhauser Comorbidity Index originally developed for mortality rather than ED use and hospitalization may have had underestimated the impact of CPI on study results $(44,46)$. Finally, findings of the study may not be generalizable to patients with MD who do not use ED, or to patients living in rural areas or in countries without a public healthcare insurance system.

\section{CONCLUSIONS}

Overall, patients with MD-CPI were more likely than those with $\mathrm{MD}$ only to experience frequent $\mathrm{ED}$ use and hospitalization for $\mathrm{MH}$ reasons. Findings also confirmed that patients with more severe medical conditions who received intensive specialized $\mathrm{MH}$ care were at greater risk for frequent $\mathrm{ED}$ use and hospitalization. Older patients were at less risk for ED use, but these patients, as well as men, were more frequently hospitalized. Higher continuity of physician care protected against frequent ED use and hospitalization. Strategies such as assertive community treatment, intensive case management, integrated co-occurring treatment, home treatment, and shared care between psychiatrists and primary care services may be implemented to improve the adequacy of care for patients with MD-CPI or for those with MD only whose clinical profiles are relatively more severe. Prevention and outreach strategies should also be strongly promoted to reduce frequent ED use and hospitalization among older age groups, and particularly among men.

\section{DATA AVAILABILITY STATEMENT}

The raw data supporting the conclusions of this article will be made available by the authors, without undue reservation.

\section{ETHICS STATEMENT}

The studies involving human participants were reviewed and approved by Douglas Mental Health Institute. Written informed consent for participation was not required for this study in accordance with the national legislation and the institutional requirements.

\section{AUTHOR CONTRIBUTIONS}

LG, M-JF, and XM designed the analytic plan for the article. LG produced the quantitative analyses and tables. LG, GG, 
and M-JF wrote the article. XM reviewed the manuscript prior to submission. All authors thus contributed to the article and approved the submitted version.

\section{FUNDING}

This study was funded by the Canadian Institutes of Health Research (CIHR, Grant Number 8400997).

\section{REFERENCES}

1. Vigod SN, Taylor VH, Fung K, Kurdyak PA. Within-hospital readmission: an indicator of readmission after discharge from psychiatric hospitalization. Can J Psychiatry. (2013) 58:476-81. doi: 10.1177/070674371305800806

2. Galarraga JE, Pines JM. Costs of ED episodes of care in the United States. Am J Emerg Med. (2016) 34:357-65. doi: 10.1016/j.ajem.2015.06.001

3. Sorup CM, Jacobsen P, Forberg JL. Evaluation of emergency department performance - a systematic review on recommended performance and quality-in-care measures. Scand J Trauma Resusc Emerg Med. (2013) 21:62. doi: 10.1186/1757-7241-21-62

4. Aaronson EL, Marsh RH, Guha M, Schuur JD, Rouhani SA. Emergency department quality and safety indicators in resourcelimited settings: an environmental survey. Int J Emerg Med. (2015) 8:39. doi: $10.1186 /$ s12245-015-0088-x

5. Fleury MJ, Fortin M, Rochette L, Grenier G, Huỳnh C, Pelletier E, et al. Assessing quality indicators related to mental health emergency room utilization. BMC Emerg Med. (2019) 19:8. doi: 10.1186/s12873-019-0223-8

6. Chwastiak LA, Davydow DS, McKibbin CL, Schur E, Burley M, McDonell MG, et al. The effect of serious mental illness on the risk of rehospitalization among patients with diabetes. Psychosomatics. (2014) 55:134-43. doi: 10.1016/j.psym.2013.08.012

7. Fleury MJ, Rochette L, Grenier G, Huynh C, Vasiliadis HM, Pelletier E, et al. Factors associated with emergency department use for mental health reasons among low, moderate and high users. Gen Hosp Psychiatry. (2019) 60:111-9. doi: 10.1016/j.genhosppsych.2019.07.006

8. Gentil L, Grenier G, Vasiliadis HM, Huynh C, Fleury MJ. Predictors of recurrent high emergency department use among patients with mental disorders. Int $J$ Environ Res Public Health. (2021) 18:4559. doi: 10.3390/ijerph18094559

9. Slankamenac K, Heidelberger R, Keller DI. Prediction of recurrent emergency department visits in patients with mental disorders. Front Psychiatry. (2020) 11:48. doi: 10.3389/fpsyt.2020.00048

10. Mark TL, Tomic KS, Kowlessar N, Chu BC, Vandivort-Warren R, Smith S. Hospital readmission among medicaid patients with an index hospitalization for mental and/or substance use disorder. J Behav Health Serv Res. (2013) 40:207-21. doi: 10.1007/s11414-013-9323-5

11. Gentil L, Grenier G, Fleury MJ. Factors related to 30-day readmission following hospitalization for any medical reason among patients with mental disorders: facteurs lies a la rehospitalisation a 30 jours suivant une hospitalisation pour une raison medicale chez des patients souffrant de troubles mentaux. Can J Psychiatry. (2021) 66:43-55. doi: 10.1177/0706743720963905

12. Chiu YM, Vanasse A, Courteau J, Chouinard MC, Dubois MF, Dubuc $\mathrm{N}$, et al. Persistent frequent emergency department users with chronic conditions: a population-based cohort study. PLoS ONE. (2020) 15:e229022. doi: 10.1371/journal.pone.0229022

13. Sarkies M, Long JC, Pomare C, Wu W, Clay-Williams R, Nguyen HM, et al. Avoiding unnecessary hospitalisation for patients with chronic conditions: a systematic review of implementation determinants for hospital avoidance programmes. Implement Sci. (2020) 15:91. doi: 10.1186/s13012-020-01049-0

14. Brennan JJ, Chan TC, Hsia RY, Wilson MP, Castillo EM. Emergency department utilization among frequent users with psychiatric visits. Acad Emerg Med. (2014) 21:1015-22. doi: 10.1111/acem.12453

\section{ACKNOWLEDGMENTS}

We gratefully acknowledged the support of the Canadian Institutes of Health Research (CIHR) and the Réseau de recherche en santé des population du Québec. We would also like to thank the Fonds de la recherche en santé du Québec (FRQ-S) for awarding a postdoctoral fellowship to the LG. Finally, we would like to thank Judith Sabetti for editorial assistance.

15. Razzano LA, Cook JA, Yost C, Jonikas JA, Swarbrick MA, Carter TM, et al. Factors associated with co-occurring medical conditions among adults with serious mental disorders. Schizophr Res. (2015) 161:45864. doi: 10.1016/j.schres.2014.11.021

16. Goldman ML, Mangurian C, Corbeil T, Wall MM, Tang F, Haselden M, et al. Medical comorbid diagnoses among adult psychiatric inpatients. Gen Hosp Psychiatry. (2020) 66:16-23. doi: 10.1016/j.genhosppsych.2020.06.010

17. Sprah L, Dernovsek MZ, Wahlbeck K, Haaramo P. Psychiatric readmissions and their association with physical comorbidity: a systematic literature review. BMC Psychiatry. (2017) 17:2. doi: 10.1186/s12888-016-1172-3

18. Simunovic Filipcic I, Filipcic I, Glamuzina L, Devcic S, Bajic Z, Bras M, et al. The effect of chronic physical illnesses on psychiatric hospital admission in patients with recurrent major depression. Psychiatry Res. (2019) 272:6028. doi: 10.1016/j.psychres.2018.12.178

19. Walker ER. Druss BG. Cumulative burden of comorbid mental disorders, substance use disorders, chronic medical conditions, and poverty on health among adults in the USA. Psychol Health Med. (2017) 22:72735. doi: 10.1080/13548506.2016.1227855

20. Bhalla IP, Rosenheck RA. A change in perspective: from dual diagnosis to multimorbidity. Psychiatr Serv. (2018) 69:1126. doi: 10.1176/appi.ps.201700194

21. Prince M, Patel V, Saxena S, Maj M, Maselko J, Phillips $\mathrm{MR}$, et al. No health without mental health. Lancet. (2007) 370:859-77. doi: 10.1016/S0140-6736(07)61238-0

22. Morden NE, Mistler LA, Weeks WB, Bartels SJ. Health care for patients with serious mental illness: family medicine's role. J Am Board Fam Med. (2009) 22:187-95. doi: 10.3122/jabfm.2009.02.080059

23. Mitchell AJ, Lord O, Malone D. Differences in the prescribing of medication for physical disorders in individuals with vs. without mental illness: metaanalysis. Br J Psychiatry. (2012) 201:435-43. doi: 10.1192/bjp.bp.111.094532

24. Gaulin M, Simard M, Candas B, Lesage A, Sirois C. Combined impacts of multimorbidity and mental disorders on frequent emergency department visits: a retrospective cohort study in Quebec, Canada. CMAJ. (2019) 191:E724-32. doi: 10.1503/cmaj.181712

25. Lidon-Moyano C, Wiebe D, Gruenewald P, Cerda M, Brown P, GoldmanMellor S. Associations between self-harm and chronic disease among adolescents: Cohort study using statewide emergency department data. $J$ Adolesc. (2019) 72:132-40. doi: 10.1016/j.adolescence.2019.03.005

26. Chwastiak LA, Rosenheck RA, Kazis LE. Utilization of primary care by veterans with psychiatric illness in the National Department of Veterans Affairs Health Care System. J Gen Intern Med. (2008) 23:183540. doi: 10.1007/s11606-008-0786-7

27. Fleury MJ, Farand L, Aube D, Imboua A. Management of mental health problems by general practitioners in Quebec. Can Fam Physician. (2012); 58:e732-8, e725-31.

28. Ross LE, Vigod S, Wishart J, Waese M, Spence JD, Oliver J, et al. Barriers and facilitators to primary care for people with mental health and/or substance use issues: a qualitative study. BMC Fam Pract. (2015) 16:135. doi: 10.1186/s12875-015-0353-3

29. Corrigan PW, Mittal D, Reaves CM, Haynes TF, Han X, Morris S, et al. Mental health stigma and primary health care decisions. Psychiatry Res. (2014) 218:35-8. doi: 10.1016/j.psychres.2014.04.028

30. Castillejos Anguiano MC, Martin Perez C, Bordallo Aragon A, Sepulveda Munoz J, Moreno Kustner B. Patterns of primary care among persons with 
schizophrenia: the role of patients, general practitioners and centre factors. Int J Ment Health Syst. (2020) 14:82. doi: 10.1186/s13033-020-00409-z

31. Kurdyak P, Vigod S, Duchen R, Jacob B, Stukel T, Kiran T. Diabetes quality of care and outcomes: comparison of individuals with and without schizophrenia. Gen Hosp Psychiatry. (2017) 46:7-13. doi: 10.1016/j.genhosppsych.2017.02.001

32. Mausbach BT, Decastro G, Schwab RB, Tiamson-Kassab M, Irwin SA. Healthcare use and costs in adult cancer patients with anxiety and depression. Depress Anxiety. (2020) 37:908-15. doi: 10.1002/da.23059

33. Shim RS, Druss BG, Zhang S, Kim G, Oderinde A, Shoyinka $S$, et al. Emergency department utilization among Medicaid beneficiaries with schizophrenia and diabetes: the consequences of increasing medical complexity. Schizophr Res. (2014) 152:4907. doi: $10.1016 /$ j.schres.2013.12.002

34. Minen MT, Tanev K. Influence of psychiatric comorbidities in migraineurs in the emergency department. Gen Hosp Psychiatry. (2014) 36:5338. doi: 10.1016/j.genhosppsych.2014.05.004

35. Albrecht JS, Hirshon JM, Goldberg R, Langenberg P, Day HR, Morgan DJ, et al. Serious mental illness and acute hospital readmission in diabetic patients. Am J Med Qual. (2012) 27:503-8. doi: 10.1177/1062860612436576

36. Sajatovic M, Welter E, Tatsuoka C, Perzynski AT, Einstadter D. Electronic medical record analysis of emergency room visits and hospitalizations in individuals with epilepsy and mental illness comorbidity. Epilepsy Behav. (2015) 50:55-60. doi: 10.1016/j.yebeh.2015.05.016

37. Bowen M, Marwick S, Marshall T, Saunders K, Burwood S, Yahyouche A, et al. Multimorbidity and emergency department visits by a homeless population: a database study in specialist general practice. Br J Gen Pract. (2019) 69:e51525. doi: 10.3399/bjgp19X704609

38. Shen C, Sambamoorthi U, Rust G. Co-occurring mental illness and health care utilization and expenditures in adults with obesity and chronic physical illness. Dis Manag. (2008) 11:153-60. doi: 10.1089/dis.2007.0012

39. Sartorius N. Comorbidity of mental and physical disorders: a key problem for medicine in the 21st century. Acta Psychiatr Scand. (2018) 137:36970. doi: $10.1111 /$ acps. 12888

40. Quebec Ministère de la Santé et des Services Sociaux. Projet de loi no 10 (2015, chapitre 1). In: Loi Modifiant l'Organisation et la Gouvernance du Réseau de la Santé et des Services Sociaux Notamment par l'Abolition des Agences Régionales. Quebec Ministère de la Santé et des Services Sociaux (2015).

41. Vérificateur Général du Québec. Rapport du Vérificateur Général du Québec à l'Assemblée Nationale pour l'Année 2015-2016. Vérification de l'Optimisation des Ressources, Automne 2015. Québec, QC: Vérificateur Général du Québec (2015).

42. Hermann RC, Rollins CK, Chan JA. Risk-adjusting outcomes of mental health and substance-related care: a review of the literature. Harv Rev Psychiatry. (2007) 15:52-69. doi: 10.1080/10673220701307596

43. Blais C, Jean S, Sirois C, Rochette L, Plante C, Larocque I, et al. Quebec Integrated Chronic Disease Surveillance System (QICDSS), an innovative approach. Chronic Dis Inj Can. (2014) 34:226-35. doi: 10.24095/hpcdp.34.4.06

44. Quan H, Sundararajan V, Halfon P, Fong A, Burnand B, Luthi JC, et al. Coding algorithms for defining comorbidities in ICD-9-CM and ICD-10 administrative data. Med Care. (2005) 43:1130-9. doi: 10.1097/01.mlr.0000182534.19832.83

45. Simard M, Dubé M, Myles G, Sirois C. La Prévalence de la Multimorbidité au Québec: Portrait Pour l'Année 2016-2017. Québec,QC: Institut national de santé publique du Québec (2019).

46. Simard M, Sirois C, Candas B. Validation of the combined Comorbidity Index of Charlson and Elixhauser to predict 30-day mortality across ICD-9 and ICD-10. Med Care. (2018) 56:441-7. doi: 10.1097/MLR.0000000000000905

47. Tu K, Mitiku T, Lee DS, Guo H, Tu JV. Validation of physician billing and hospitalization data to identify patients with ischemic heart disease using data from the Electronic Medical Record Administrative data Linked Database (EMRALD). Canad J Cardiol. (2010) 26:e225e8. doi: 10.1016/s0828-282x(10)70412-8

48. Weisman A, Tu K, Young J, Kumar M, Austin PC, Jaakkimainen L, et al. Validation of a type 1 diabetes algorithm using electronic medical records and administrative healthcare data to study the population incidence and prevalence of type 1 diabetes in Ontario, Canada. BMJ Open Diabetes Res Care. (2020) 8:e001224. doi: 10.1136/bmjdrc-2020-001224
49. Pampalon R, Hamel D, Gamache P, Raymond G. A deprivation index for health planning in Canada. Chronic Dis Can. (2009) 29:178-91. doi: 10.24095/hpcdp.29.4.05

50. Rodriguez HP, Marshall RE, Rogers WH, Safran DG. Primary care physician visit continuity: a comparison of patient-reported and administratively derived measures. J Gen Intern Med. (2008) 23:1499-502. doi: 10.1007/s11606-008-0692-z

51. Dreiher J, Comaneshter DS, Rosenbluth Y, Battat E, Bitterman H, Cohen $\mathrm{AD}$. The association between continuity of care in the community and health outcomes: a population-based study. Isr J Health Policy Res. (2012) 1:21. doi: 10.1186/2045-4015-1-21

52. Breslau N, Reeb KG. Continuity of care in a university-based practice. J Med Educ. (1975) 50:965-9. doi: 10.1097/00001888-197510000-00006

53. Romaire MA, Haber SG, Wensky SG, McCall N. Primary care and specialty providers: an assessment of continuity of care, utilization, and expenditures. Med Care. (2014) 52:1042-9. doi: 10.1097/MLR.0000000000000246

54. Bartlett Foote S. Coexistence, conflict, and cooperation: public policies toward medical devices. J Health Polit Policy Law. (1986) 11:501-23.

55. Huynh C, Ferland F, Blanchette-Martin N, Menard JM, Fleury MJ. Factors influencing the frequency of emergency department utilization by individuals with substance use disorders. Psychiatr Q. (2016) 87:71328. doi: 10.1007/s11126-016-9422-6

56. Vu F, Daeppen JB, Hugli O, Iglesias K, Stucki S, Paroz S, et al. Screening of mental health and substance users in frequent users of a general Swiss emergency department. BMC Emerg Med. (2015) 15:27. doi: 10.1186/s12873-015-0053-2

57. Coulson C, Ng F, Geertsema M, Dodd S, Berk M. Client-reported reasons for non-engagement in drug and alcohol treatment. Drug Alcohol Rev. (2009) 28:372-8. doi: 10.1111/j.1465-3362.2009.00054.x

58. Brorson HH, Ajo Arnevik E, Rand-Hendriksen K. Duckert F. Drop-out from addiction treatment: a systematic review of risk factors. Clin Psychol Rev. (2013) 33:1010-24. doi: 10.1016/j.cpr.2013.07.007

59. Penzenstadler L, Gentil L, Huynh C, Grenier G, Fleury MJ. Variables associated with low, moderate and high emergency department use among patients with substance-related disorders. Drug Alcohol Depend. (2020) 207:107817. doi: 10.1016/j.drugalcdep.2019.107817

60. Germack HD, Caron A, Solomon R, Hanrahan NP. Medical-surgical readmissions in patients with co-occurring serious mental illness: a systematic review and meta-analysis. Gen Hosp Psychiatry. (2018) 55:6571. doi: 10.1016/j.genhosppsych.2018.09.005

61. De Hert M, Cohen D, Bobes J, Cetkovich-Bakmas M, Leucht S, Ndetei $\mathrm{DM}$, et al. Physical illness in patients with severe mental disorders. II Barriers to care, monitoring and treatment guidelines, plus recommendations at the system and individual level. World Psychiatry. (2011) 10:13851. doi: 10.1002/j.2051-5545.2011.tb00036.x

62. Lichstein JC, Domino ME, Beadles CA, Ellis AR, Farley JF, Morrissey JP, et al. Use of medical homes by patients with comorbid physical and severe mental illness. Med Care. (2014) 52(Suppl 3):S85-91. doi: 10.1097/MLR.0000000000000025

63. Penzenstadler L, Gentil L, Grenier G, Khazaal Y, Fleury MJ. Risk factors of hospitalization for any medical condition among patients with prior emergency department visits for mental health conditions. BMC Psychiatry. (2020) 20:431. doi: 10.1186/s12888-020-02835-2

64. Bodner E, Cohen-Fridel S, Iancu I. Staff attitudes toward patients with borderline personality disorder. Compr Psychiatry. (2011) 52:54855. doi: 10.1016/j.comppsych.2010.10.004

65. Woollaston K, Hixenbaugh P. Destructive Whirlwind: nurses' perceptions of patients diagnosed with borderline personality disorder. I Psychiatr Ment Health Nurs. (2008) 15:703-9. doi: 10.1111/j.1365-2850.2008. 01275.x

66. Links PS, Ross J, Gunderson JG. Promoting good psychiatric management for patients with borderline personality disorder. J Clin Psychol. (2015) 71:75363. doi: $10.1002 /$ jclp. 22203

67. Choi-Kain LW, Finch EF, Masland SR, Jenkins JA, Unruh BT. What works in the treatment of borderline personality disorder. Curr Behav Neurosci Rep. (2017) 4:21-30. doi: 10.1007/s40473-017-0103-Z

68. Bateman AW, Gunderson J, Mulder R. Treatment of personality disorder. Lancet. (2015) 385:735-43. doi: 10.1016/S0140-6736(14)61394-5 
69. Combs H, Markman J. Anxiety disorders in primary care. Med Clin North Am. (2014) 98:1007-23. doi: 10.1016/j.mcna.2014.06.003

70. Cameron C, Habert J, Anand L, Furtado M. Optimizing the management of depression: primary care experience. Psychiatry Res. (2014) 220(Suppl 1):S45-57. doi: 10.1016/S0165-1781(14)70005-8

71. Loranger C, Fleury MJ. Factors associated with perceived continuity of care among patients suffering from mental disorders. Community Ment Health J. (2020) 56:670-9. doi: 10.1007/s10597-019-00528-z

72. Biringer E, Hartveit M, Sundfor B, Ruud T, Borg M. Continuity of care as experienced by mental health service users - a qualitative study. BMC Health Serv Res. (2017) 17:763. doi: 10.1186/s12913-017-2719-9

73. Singh P, Chakravarthy B, Yoon J, Snowden L, Bruckner TA. Psychiatricrelated revisits to the emergency department following rapid expansion of community mental health services. Acad Emerg Med. (2019) 26:133645. doi: $10.1111 /$ acem. 13812

74. Mitton CR, Adair CE, McDougall GM, Marcoux G. Continuity of care and health care costs among persons with severe mental illness. Psychiatr Serv. (2005) 56:1070-6. doi: 10.1176/appi.ps.56.9.1070

75. Ride J, Kasteridis P, Gutacker N, Doran T, Rice N, Gravelle H, et al. Impact of family practice continuity of care on unplanned hospital use for people with serious mental illness. Health Serv Res. (2019) 54:131625. doi: 10.1111/1475-6773.13211

76. Jacobs R, Aylott L, Dare C, Doran T, Gilbody S, Goddard M, et al. The Association Between Primary Care Quality and Health-Care Use, Costs and Outcomes for People With Serious Mental Illness: A Retrospective Observational Study. Southampton: Health Services and Delivery Research (2020).

77. Byers AL, Arean PA, Yaffe K. Low use of mental health services among older Americans with mood and anxiety disorders. Psychiatr Serv. (2012) 63:66-72. doi: 10.1176/appi.ps.201100121

78. Barry LC, Abou JJ, Simen AA, Gill TM. Under-treatment of depression in older persons. J Affect Disord. (2012) 136:78996. doi: 10.1016/j.jad.2011.09.038
79. Heflinger CA, Hinshaw SP. Stigma in child and adolescent mental health services research: understanding professional and institutional stigmatization of youth with mental health problems and their families. Adm Policy Ment Health. (2010) 37:61-70. doi: 10.1007/s10488-010-0294-z

80. Plaistow J, Masson K, Koch D, Wilson J, Stark RM, Jones PB, et al. Young people's views of UK mental health services. Early Interv Psychiatry. (2014) 8:12-23. doi: 10.1111/eip.12060

81. Affleck W, Carmichael V, Whitley R. Men's mental health: social determinants and implications for services. Can J Psychiatry. (2018) 63:5819. doi: $10.1177 / 0706743718762388$

82. Farrer LM, Walker J, Harrison C, Banfield M. Primary care access for mental illness in Australia: patterns of access to general practice from 2006 to 2016. PLoS ONE. (2018) 13:e0198400. doi: 10.1371/journal.pone.0198400

Conflict of Interest: The authors declare that the research was conducted in the absence of any commercial or financial relationships that could be construed as a potential conflict of interest.

Publisher's Note: All claims expressed in this article are solely those of the authors and do not necessarily represent those of their affiliated organizations, or those of the publisher, the editors and the reviewers. Any product that may be evaluated in this article, or claim that may be made by its manufacturer, is not guaranteed or endorsed by the publisher.

Copyright $\odot 2021$ Gentil, Grenier, Meng and Fleury. This is an open-access article distributed under the terms of the Creative Commons Attribution License (CC BY). The use, distribution or reproduction in other forums is permitted, provided the original author(s) and the copyright owner(s) are credited and that the original publication in this journal is cited, in accordance with accepted academic practice. No use, distribution or reproduction is permitted which does not comply with these terms. 